\title{
Estudo comparativo experimental da injeção perineural do álcool benzílico $0,75 \%$ e do álcool etílico absoluto nos nervos palmares de eqüinos
}

José Luiz de Mello NICOLETTI ${ }^{1}$

Pierre Barnabé ESCODRO ${ }^{1}$

Carlos Alberto HUSSNI ${ }^{1}$

Ana Liz Garcia ALVES ${ }^{1}$

Armen THOMASSIAN ${ }^{1}$

René Laufer AMORIN²

\section{Correspondência para:}

Distrito de Rubião Júnior CEP 18618-000 -

Fone (014) 3811-6252

e-mail: nicoletti@fmvz.unesp.br

Recebido para publicação: 20/03/2005 Aprovado para publicação: 24/05/2007

\begin{abstract}
1 - Departamento de Cirurgia e Anestesiologia Veterinária da Faculdade de Medicina Veterinária e Zootecnia da Universidade Estadual Paulista, Botucatu-SP 2 - Departamento de Clínica Veterinária da Faculdade de Medicina Veterinária e Zootecnia da Universidade Estadual Paulista, Botucatu-SP
\end{abstract}

\begin{abstract}
Resumo
Foi estudada a neurólise química dos nervos Palmares em 10 eqüinos. Usando-se uma ferradura especial para induzir claudicação, a locomoção avaliada ao passo e trote e os graus foram registrados antes e após a injeção perineural do álcool benzílico 0,75\% (Grupo A) e o álcool etílico absoluto (Grupo B). Os testes mostraram que ambos os neurolíticos dessensibilizaram completamente a sola dos animais a partir de 30 dias, assim permanecendo até o $150^{\circ}$ dia nos animais do Grupo A, quando ocorreu retorno progressivo da sensibilidade. Já no Grupo B, aos 180 dias, a sensibilidade permanecia ausente. A análise histopatológica revelou que nos nervos infiltrados com álcool benzílico $0,75 \%$ prevaleceram lesões próprias de axonotemese, sugerindo tendência de regeneração e restabelecimento da condução nervosa, enquanto que nos nervos infiltrados com álcool etílico absoluto, prevaleceram lesões simuladoras de neurotemese, com degeneração nervosa permanente. Concluiu-se que os álcoois utilizados foram eficientes bloqueadores temporários ou permanentes dos nervos Palmares em eqüinos.
\end{abstract}

\section{Introdução}

Apesar do avanço científico e tecnológico, a dor, em suas diferentes formas de manifestação, continua sendo um dos maiores problemas médicos afetando milhões de pessoas em todo mundo, e a terapia antiálgica, interrompendo as vias de transmissão nervosa, visa aliviar a dor de diferentes tipos, origens e intensidades, beneficiando, sobretudo pacientes com câncer terminal. ${ }^{1,2,3,4}$

Muitos procedimentos antiálgicos em humanos podem ser aplicados aos animais, porém existem poucos estudos controlados sobre o assunto na medicina veterinária. Merkens e Schamhardt ${ }^{5}$ induziram claudicação em eqüinos com dois parafusos de $25 \mathrm{~mm}$ de comprimento e $8 \mathrm{~mm}$ de diâmetro na ferradura para produzir pressão controlada na sola, que serviu de modelo para outras pesquisas. ${ }^{6,7,8,9}$

Os neurolíticos bloqueiam temporária ou permanentemente a transmissão nervosa, sendo indicados para dor crônica refratária ao tratamento conservativo, preconizandoos caso haja alívio da dor com o bloqueio anestésico prévio. ${ }^{10,11,12} \mathrm{Em}$ eqüinos, um terço das claudicações se devem a dor crônica na metade palmar do pé, e melhoram ou são eliminadas com o bloqueio do nervo digital palmar. ${ }^{13}$

Existem três tipos de lesões nervosas periféricas, conforme a gravidade: na neuropraxia há perda da condução dos impulsos nervosos, porém sem degeneração estrutural do nervo, permitindo recuperar a função; na axonotmese embora ocorra degeneração walleriana, estão preservados os tubos endoneurais (epineuro e perineuro) e células de Schwann, permitindo a regeneração da função nervosa; a 
neurotmese é a lesão mais grave, ocorrendo descontinuidade e destruição dos elementos neurais constituídos pelo epineuro, perineuro e endoneuro, além das fibras nervosas, dificultando ou impossibilitanto a regeneração dos axônios. ${ }^{14,15}$

Os neurolíticos lesam as fibras nervosas dependendo da concentração do agente em contato com o tecido. As injeções intra ou perineurais produzem alterações axonais importantes e degeneração walleriana. ${ }^{16,17}$

O álcool etílico ou etanol atua extraindo os fosfolipídeos e colesterol do tecido nervoso, precipitando as lipo e mucoproteínas, e quando injetado diretamente no nervo, bloqueia a condução nervosa, causando anestesia ou hipoestesia e paralisia, se afetar os nervos motores. É irritante, causa dor no local da injeção e tem ampla difusão nos tecidos. Em nervos periféricos, o álcool etílico lesa as células de Schwann, interrompendo a transmissão nervosa na bainha de mielina e causando degeneração walleriana, e a dor é abolida até serem restauradas as fibras nervosas e a transmissão normal de impulsos, porém às vezes, a destruição do nervo é permanente. ${ }^{16,17,18,19,20,21} \mathrm{O}$ álcool etílico pode ainda causar fibrose local e dificultar uma subseqüente neurectomia cirúrgica. ${ }^{22,23}$

O álcool benzílico a $0,75 \%$ (SarapinÒ) é um neurolítico alcalóide de uso humano, indicado na síndroma dolorosa do nervo ciático, neuralgia intercostal, neurites, neuralgia lombar e do nervo trigêmeo, entre outras. Em animais, seu uso mais comum tem sido para ablação química do nervo Digital Palmar em eqüinos com enfermidades crônicas do pé, porém, existem divergências quanto ao tempo de ação da droga, bem como são desconhecidas as alterações histopatológicas no nervo injetado.

Este trabalho comparou o tempo de ação analgésica e aspectos histopatológicos provocados pela injeção perineural do álcool benzílico $0,75 \%$ e do álcool etílico absoluto nos nervos palmares de eqüinos sadios, utilizando-se como modelo experimental ferradura projetada para induzir dor na sola.

\section{Material e Método}

Nove eqüinos da raça Puro Sangue Inglês e um da raça Mangalarga Marchador, pesando entre 380 e $450 \mathrm{~kg}$ de ambos os sexos, hígidos, foram distribuídos em dois grupos de cinco animais. Em todos os animais, foi colocada ferradura padrão no membro torácico direito (MTD), enquanto que no membro torácico esquerdo (MTE), foi aplicada ferradura especial com duas barras e cinco orifícios com rosca para parafusos de $6 \mathrm{~mm}$ de diâmetro e comprimentos de $11 \mathrm{~mm}$ (ponto p), $15 \mathrm{~mm}$ (pontos ms-ls) e $18 \mathrm{~mm}$ (pontos r-s), conforme a figura 1 .

Após uma semana de adaptação, os

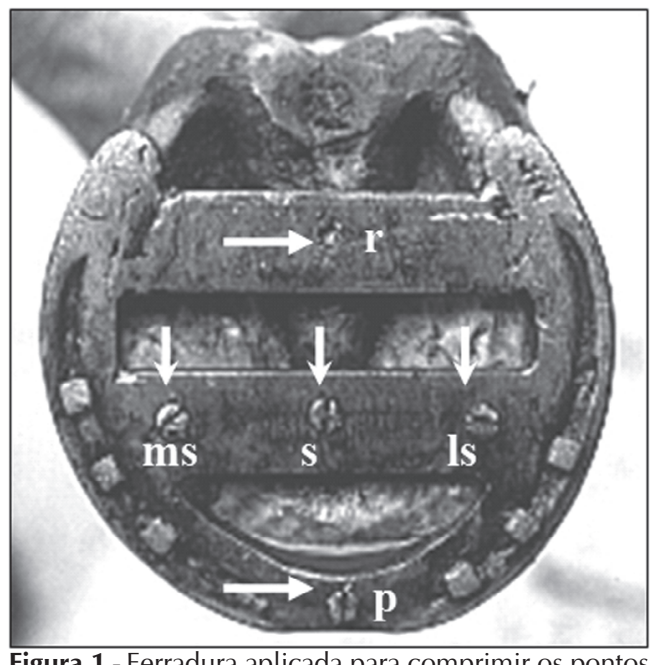

Figura 1 - Ferradura aplicada para comprimir os pontos $\mathbf{p}, \mathbf{m s}$, Is, r, s na sola e ranilha

parafusos da ferradura do MTE foram apertados com chave de fenda, pressionando um ponto de cada vez, até o animal mostrar claudicação de apoio, mostrada a passo e trote, e classificada em graus de 1 a $4 .{ }^{24}$ Após registrar em protocolo individual o grau de claudicação apresentado em cada ponto, manifestado a passo e trote, a pressão do parafuso foi aliviada, até restabelecer a normalidade locomotora (Grau 0).

Nos animais do Grupo A foi aplicada infiltração perineural com $5 \mathrm{ml}$ de álcool benzílico 0,75\% (Sarapin - Produto composto de extrato de planta Sarraceniacea. 
High Chemical Company -EUA) no nervo Palmar do MTE, entre o ligamento suspensor do boleto e o tendão flexor digital profundo, e nos animais do Grupo B, seguindo os mesmos procedimentos, foi injetado $5 \mathrm{ml}$ de álcool etílico absoluto (Álcool Etílico Absoluto - Produto de manipulação farmacêutica-Farmácia da USP). Doze horas após a infiltração, os parafusos foram apertados um de cada vez, e reavaliados e registrados os graus de claudicação em cada ponto. Concluída a avaliação, os parafusos foram removidos na mesma seqüência até as avaliações subseqüentes, realizadas com $24,48,72$ horas, sete dias e, então, mensalmente, até um eventual retorno da sensibilidade na região da sola.

Finda a avaliação, segmentos de aproximadamente $10 \mathrm{~cm}$ dos nervos Palmares infiltrados foram colhidos cirurgicamente para exame histopatológico, processados em cortes transversais e longitudinais de $3 \mu \mathrm{com}$ coloração pela Hematoxilina Eosina e Luxol fast blue para a bainha de mielina.

A classificação das alterações observadas no exame histopatológico em tecido nervoso foi baseada nas citações de Adair e Andrews ${ }^{14}$ e Quan e Bird ${ }^{15}$.

\section{Resultados}

A ferradura para induzir claudicação, as medidas dos parafusos e a pressão aplicada nos pontos ms, s, $\mathbf{l s ,} \mathbf{p}, \mathbf{r}$, causou imediata claudicação de apoio de Graus 2 ou 3, em todos os animais de ambos os

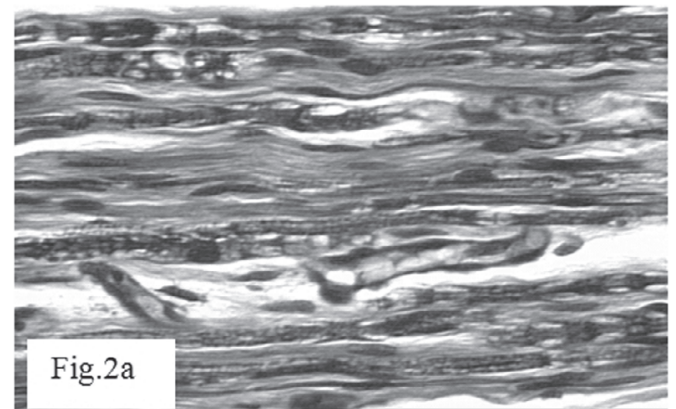

Figura 2a - Degeneração walleriana. Axonotemese. Luxol fast blue $-640 \mathrm{X}$ grupos. Até 72 horas da infiltração dos nervos Palmares com álcool benzílico 0,75\% $\left(\right.$ Sarapin $\left.{ }^{\circledR}\right)$ - Grupo A, os animais apresentaram claudicação de Graus 2 ou 3 nos mesmos pontos testados. A partir do $7^{\circ}$ dia, a sensibilidade regrediu para Graus 1 ou 2, até a perda total da sensibilidade (Grau 0) , que persistiu dos 30 dias até 120 a 150 dias. A partir de 150 dias, houve retorno gradativo da sensibilidade aos testes de pressão nos pontos $\mathbf{m s}, \mathbf{s}, \mathbf{1 s}, \mathbf{r}$, manifestada por claudicação de Graus 1 ou 2.

Nas primeiras 24 horas após a injeção perineural do álcool etílico absoluto - Grupo $\mathrm{B}$, todos os animais apresentaram reação inflamatória local, com edema na região metacárpica e claudicação leve a moderada, o que retardou a avaliação clínica dos animais por 48 horas. O edema diminuiu gradativamente até desaparecer completamente entre três a quatro semanas. Após 48 horas da infiltração, os testes de sensibilidade mostraram claudicação de Graus 1 ou 2, desaparecendo com 72 horas (Grau 0) e assim permanecendo até o último teste realizado aos 180 dias.

Ao exame histopatológico, observouse nos nervos palmares infiltrados com álcool benzílico $0,75 \%$ (Sarapin $\left.{ }^{\circledR}\right)$ (Grupo A) áreas focais ou difusas de necrose, degeneração walleriana das fibras nervosas remanescentes, infiltrado inflamatório mononuclear perivascular e presença de esferóides (Figuras 2a e 2b).

Os nervos infiltrados com álcool etílico absoluto (Grupo B) mostraram infiltrado inflamatório mononuclear, necrose de feixes nervosos, degeneração walleriana

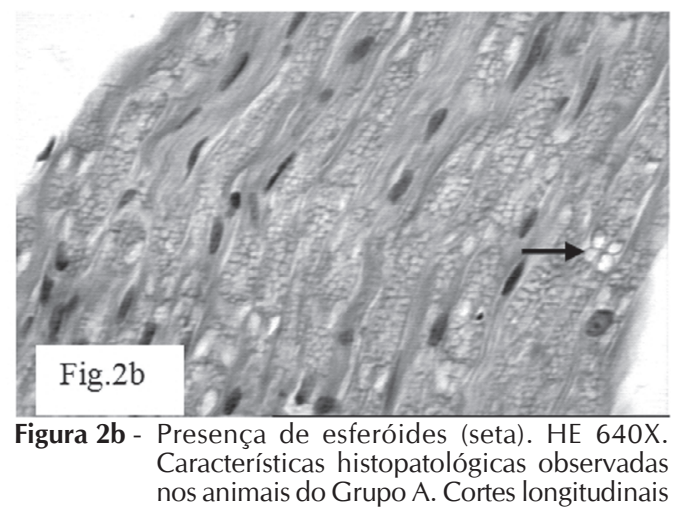




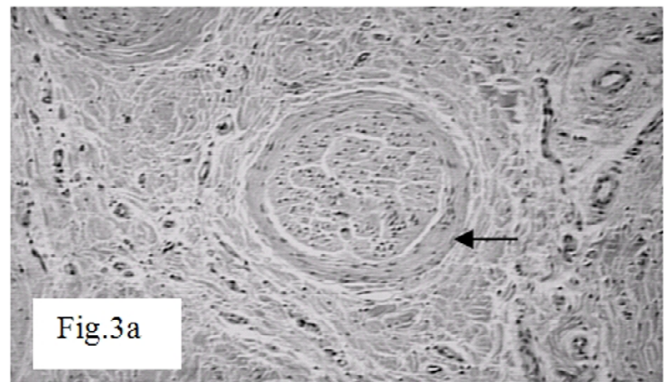

Figura 3a-Infiltrado inflamatório mononuclear. Espessamento do perineuro (seta). HE 100X

e espessamento do perineuro (Figuras 3a e $3 b)$.

\section{Discussão e Conclusões}

A ferradura utilizada para induzir claudicação no presente experimento foi adaptação da idealizada por Merkens e Schamhardt ${ }^{5}$, pois além dos dois parafusos posicionados na sola, acrescentou-se um outro na pinça, no ápice e no sulco central da ranilha. A claudicação induzida foi semelhante aos demais modelos baseados no mesmo princípio, o que favorece a indicação do modelo mais simples utilizado por outros autores, pela simplicidade de confecção e número menor de pontos de pressão a serem testados. ${ }^{7,89}$

A intensidade da dor produzida na sola foi proporcional à pressão aplicada nos parafusos, podendo ser controlada de acordo com o grau de claudicação desejado. Nesta pesquisa, conforme o protocolo estipulado, as dimensões dos parafusos e a pressão aplicada deveriam induzir claudicação evidenciada clinicamente, com características de Graus 2 ou 3, segundo a classificação de Stashak. ${ }^{24}$

A claudicação manifestada reduziu gradativamente de Grau 3 ou 2 até ser abolida totalmente após a infiltração perineural tanto do alcool benzílico 0,75\% quanto do álcool etílico absoluto.

No Grupo A, a sensibilidade da sola retornou à partir do $150^{\circ}$ dia após a infiltração do neurolítico, enquanto que no Grupo B, a mesma permaneceu ausente aos 180 dias após a infiltração.

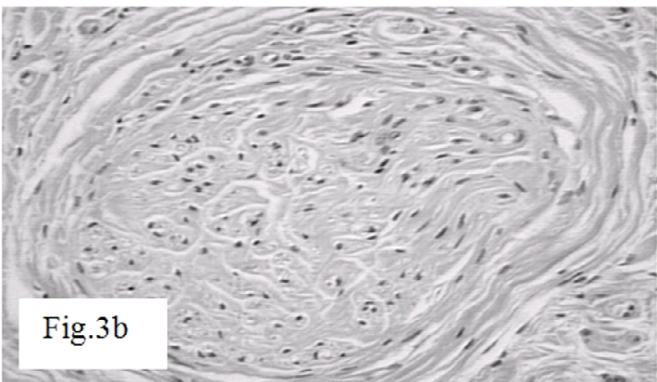

Figura 3b - Necrose do nervo. HE 400X. Características histopatológicas observadas nos animais do Grupo B. Cortes transversais

Sabe-se que a citotoxicidade de um neurolítico está diretamente relacionada à concentração utilizada e o local de deposição do mesmo. ${ }^{16,17}$ A infiltração extrafascicular causa menor dano ao nervo, caracterizado por edema no endoneuro, inflamação e lesão na bainha de mielina e células de Schwann, enquanto a infiltração intrafascicular é muito mais lesiva, causando geralmente degeneração walleriana. ${ }^{2,18,20,21}$ A concentração do neurolítico e o local preciso de sua deposição em relação ao nervo podem justificar as reações locais e as variações das lesões histopatológicas observadas, que definem a extensão da degeneração nervosa.

Estudos no homem, apontam que a injeção da solução neurolítica em local incorreto, é uma das maiores complicações imediatas decorrentes da neurólise química. ${ }^{2}$ Nesta pesquisa, exceto a celulite transitória no local infiltrado com álcool etílico absoluto, atribuído à ação irritante do mesmo em alta concentração, não ocorreram outras complicações secundárias à neurólise com as soluções alcóolicas utilizadas.

A irritação no local da injeção com o álcool etílico, embora de recuperação espontânea e sem prejuízos estéticos, justifica, a nosso ver, prévia anestesia local, minutos antes de infiltrar o neurolítico, mesmo em concentrações menores, a fim de minimizar a dor. O preparo de solução contendo uma associação do neurolítico com anestésico local, no caso a bupivacaína, parece ser método prático e eficiente, como refere Dockery ${ }^{25}$.

$\mathrm{Na}$ utilização de neurolíticos 
alcoólicos em altas concentrações, deve-se sempre considerar a possibilidade da difusão inadvertida do produto aos tecidos ou nervos vizinhos, podendo causar déficit motor, dor, celulite ou formação de abscessos, como advertem outros pesquisadores. ${ }^{2,4,20}$

A ausência de reações cicatriciais nos tecidos perineurais e a aparente normalidade macroscópica dos nervos infiltrados, observadas durante a remoção cirúrgica dos mesmos para exame histopatológico, em especial no grupo infiltrado com álcool etílico absoluto, contrastam com a observação de fibrose local, referida por outros autores. ${ }^{22,23}$ Entretanto, esse risco deve ser considerado.

Injeções semanais do álcool etílico a $4 \%$ causam degeneração progressiva do nervo até a perda total da função, antes, porém, após a $1^{\mathrm{a}}$ ou $2^{\mathrm{a}}$ injeções, o álcool produz neurite transitória, que causa dor. ${ }^{6} \mathrm{~A}$ ampla variação na concentração do álcool etílico para neurólise em humanos - de 3\% ou $4 \%$ até $80 \%$ a $95 \%$, mostra indefinição quanto a concentração ideal da droga para aquela finalidade. ${ }^{6,16,18,20,21,22}$

Por ser assunto pouco explorado na espécie eqüina, os resultados desta pesquisa sugerem investigações quanto a concentração e número de injeções ideais para os melhores efeitos neurolíticos do álcool etílico ou etanol, já que o álcool benzílico é formulado na concentração padrão de $0,75 \%$ (Sarapin $\left.^{\circledR}\right)$.

A análise histopatológica dos nervos infiltrados com o álcool benzílico $0,75 \%$ e álcool etílico absoluto, mostraram os efeitos degenerativos destes neurolíticos, sendo sinais compatíveis com axonotmese ou neurotmese, conforme a classificação de Seddon (1941), citado por Quan e Bird ${ }^{15}$. Essa classificação é baseada em lesões nervosas associadas a traumas mecânicos e não por substâncias químicas. O menor efeito agressivo do álcool benzílico $0,75 \%$ resultou em degeneração nervosa do tipo axonotmese, passível portanto de regeneração, enquanto que o álcool etílico absoluto, causou degeneração que simula ser do tipo neurotmese, ou seja potencialmente permanente. ${ }^{16,17,18,19,20}$ Macroscopicamente, durante a remoção cirúrgica do material para exame histopatológico, não foi observado neuroma após neurólise alcoólica, como foi observado com outros neurolíticos em seres humanos. ${ }^{11}$

A ferradura com parafusos foi um modelo experimental eficiente e reversível para induzir claudicação, sem causar seqüela nos animais.

Baseado nos resultados, concluiu-se que a injeção perineural nos nervos Palmares com álcool benzílico 0,75\% e álcool etílico absoluto foi eficiente para dessensibilizar a superfície solear dos eqüinos, sendo que as lesões nos nervos infiltrados com álcool benzílico $0,75 \%$, foram típicas de axonotmese e nos nervos infiltrados com álcool etílico absoluto, apresentaram características clínicas que sugerem lesão do tipo neurotmese.

Agradecimentos à FAPESP pelo auxílio concedido (FAPESP 02/10314-8).

\section{Experimental comparative study of perineural injection of benzyl alcohol $0,75 \%$ and absolute ethyl alcohol in equine palmar nerves}

\begin{abstract}
The clinical and histopathological effects of two alcoholic neurolytics were studied in horses. Normal horses were shod with a designed shoe adaptted with 5 screws to produce solar pain. After gait and lameness score analysis, the palmar nerve of 5 horses was injected with $5 \mathrm{ml}$ of $0,75 \%$ benzyl alcohol (Group A) and 5 horses were
\end{abstract}

Key words: Neurolysis. Alcohol. Palmar nerves. Horse. 
injected with $5 \mathrm{ml}$ of absolute ethyl alcohol (Group B). The animals were submitted to regular lameness evaluation and solar sensibility tests during next six months. The solar sensitivity returned 5 months latter in the group injected with benzyl alcohol $0,75 \%$, while in the group injected with absolute ethyl alcohol, the sole was still desensitized 6 months latter. The histopathological findings showed that the nerve injected with benzyl alcohol $0,75 \%$, resulted in axonotmesis, characterized by axonal nerve degeneration, with possibilities for the nerve conduction recovery. The perineural injection of ethyl alcohol absolute, resulted in neurotmesis with difficult nerve regeneration. It was concluded that chemical neurolysis with alcohol is an option for temporary or permanent nerve blocks in horses.

\section{Referências}

1 ALLEN Jr, L. Compounding for the management of pain. Secundum Artem, v. 8, n. 4, p. 1-5, 2003.

2 COLES, P.; THOMPSON, G. The role of neurolytic blocks in the treatment of cancer pain. Anesthesiology Clinic, v. 29, n. 1, p. 93-104, 1991.

3 McQUAY, $\mathrm{H}$. Relief of non-malignant chronic pain. 2002. Disponível em: <http://med-lob.ru/english/ oxford/non-mal-pain.shtml > . Acesso em: 23 out. 2003.

4 RICHEIMER, S. H.; MACRES, S. M. Procedural and Medical Complications of Chronic Pain Management. 2000. Disponível em: < http://www.helpforpain.com/ articles/complications/complications.htm $>$. Acesso em: 16 dez. 2003.

5 MERKENS, H. W.; SCHAMHARDT, H. C. Evaluation of equine locomotion during different degrees of experimentally induced lameness I: Lameness model and qualification of ground reaction force patterns of the limbs. Equine Veterinary Journal, p. 99-106, 1983. Supplement 6.

6 CLARK, C. Horse lameness on the block-Specially designed horseshoe becomes teaching tool. Disponível em: < http:/www.uoguelph.ca/research/publications/ Assets/HTML_MAGS/equine/p14.html > . Acesso em: 25 out. 2003

7 SARDARI, K.; KAZEMI, H.; MOHRI, M. Effects of analgesia of the distal interphalangeal joint and navicular bursa on experimental lameness caused by solar pain in horses. Journal Veterinary Medicine Physiology Clinic Med, v. 49, n. 9, p. 478-481, 2002.

8 SCHUMACHER, J. et al. Abolition of Lameness Caused by Experimentally Induced Solar Pain in Horses after Analgesia of the Distal Interphalangeal Joint. American Association Equine Practioner, v. 45, p. 193194, 1999.

9 XIE, H.; OTT, E. A.; COLAHAN, P. Influence of Acupunture on Experimental Lameness in Horses. American Association Equine Practioner, v. 47, p. 347356, 2001.

10 FERRANTE, M. F. The Anesthesia pain management program. University of Pennsylvania health system, 2000. Disponível em: < http: // www.med.upenn.edu/ cancer htm > . Acesso em: 2 ago. 2000.

11 PORTER, B. R. Neurolysis. In: University of lowa health care. Disponível em: < http://www.uiowa.edu/ c116006/neurolysis >. Acesso em: 2 ago. 2000.

12 RYKOWSKI, J. J.; HILGIER, M. Efficacy of Neurolytic Celiac Plexus Block in varying locations of Pancreatic Cancer. Reg. Anesth, v. 92, p. 347-354, 2000.

13 TROTER, G. W. Aspects of Palmar Heel Pain. American Association Equine Practioner, v. 45, p. 195197, 1999.

14 ADAIR, H. S., ANDREWS, F. M. Diseases of the Peripheral Nerves. In: KOBLUK, C. N.; AMES, T. R.; GEOR, R. J. (Ed.). The Horse. Diseases and clinical managment. Philadelphia: W.B.Saunders, 1995. p. 473485.

15 QUAN, D.; BIRD, S. J. Nerve Conduction Studies and Electromyography in the Evaluation of Peripheral Nerve Injuries. The University of Pennsylvania Orthopedic Journal, v. 12, p. 45-51, 1999.

16 LIPTON, S. Neurolysis: pharmacology and drug selection. Care: J. B. Lippincott, 1993. p. 343-358.

17 NEIRA, F.; ORTEGA, J. L.; CARRASCO, M. S. Bloqueos neurolíticos: farmacologia y neurolisis de los nervios craneales. Revista Sociedad Espanhola Dolor v. 3, p. 191-201, 1996.

18 BOOTH, N. H.; McDONALD, L. E. Quimioterapia de Doenças Microbianas, Fúngicas e Virais. In: BOOTH, N. H.; McDONALD, L. E. Farmacologia e terapêutica em veterinária. 6 . ed. Rio de Janeiro: Guanabara Koogan, 1992. p. 617-632.

19 FERRANTE, M. F. Neurolytics procedures for cancer pain management. Online Pain Journal, 2001. Disponível em: <http:// www. pain.com/ onlinepainjournal/default.cfm >. Acesso em : 23 set. 2001.

20 MYERS, R.; KATZ, J. Agentes neurolíticos. In: RAJ, P. R. Tratamiento práctico del dolor. Mosby, 1992. p. 689-700.

21 ROWE, D. S. Neurolytic Techniques For Pain 
Management. 1998. Disponível em: <http:/ www.dcmsonline.org/jax-medicine/1998journals/ october98/neurolytic.htm > . Acesso em: 15 dez. 2003.

22 ANDREAE-JONES, S. Invasive treatment. ASAMS, 2000. Disponível em: <http:/ www.aboutarachnoiditis.org/content/invasivetreatment/invasive-treatment.ht $>$. Acesso em: $15 \mathrm{dez}$. 2003.

23 MADISON, J. B.; DYSON, S. J. Treatment and Prognosis of Horses with Navicular Disease. In: ROSS, M. W.; DYSON, S. J. Diagnosis and managment of lameness in the horse. Philadelphia: Saunders, 2003. 1140 p.

24 STASHAK,T. S. Adam 's lameness in horses. 4th ed. Philadelphia: Lea \& Febiger, 1987. p. 106

25 DOCKERY, G. L. Alcohol injection Targets intermetatarsal pain. Bio Mechanics. Pain Management. Disponível em: < http://journals.iranscience.net:800/ Default/www.biomech.com/current/pain.shtml $>$. Acesso em: 15 dez. 2003. 